\title{
The representation of the symmetric group on $m$-Tamari intervals
}

\author{
Mireille Bousquet-Mélou ${ }^{1}$ and Guillaume Chapuy ${ }^{2}$ and Louis-François \\ Préville-Ratelle ${ }^{3}$ \\ ${ }^{1}$ CNRS, LaBRI, UMR 5800, Université de Bordeaux, 351 cours de la Libération, 33405 Talence Cedex, France \\ ${ }^{2}$ CNRS, LIAFA, UMR 7089, Université Paris Diderot - Paris 7, Case 7014, 75205 Paris Cedex 13, France \\ ${ }^{3}$ LACIM, UQAM, C.P. 8888 Succ. Centre-Ville, Montréal H3C 3P8, Canada
}

\begin{abstract}
An $m$-ballot path of size $n$ is a path on the square grid consisting of north and east unit steps, starting at $(0,0)$, ending at $(m n, n)$, and never going below the line $\{x=m y\}$. The set of these paths can be equipped with a lattice structure, called the $m$-Tamari lattice and denoted by $\mathcal{T}_{n}^{(m)}$, which generalizes the usual Tamari lattice $\mathcal{T}_{n}$ obtained when $m=1$. This lattice was introduced by F. Bergeron in connection with the study of diagonally coinvariant spaces in three sets of $n$ variables. The representation of $\mathfrak{S}_{n}$ on these spaces is conjectured to be closely related to the natural representation of $\mathfrak{S}_{n}$ on (labelled) intervals of the $m$-Tamari lattice studied in this paper.

An interval $[P, Q]$ of $\mathcal{T}_{n}^{(m)}$ is labelled if the north steps of $Q$ are labelled from 1 to $n$ in such a way the labels increase along any sequence of consecutive north steps. The symmetric group $\mathfrak{S}_{n}$ acts on labelled intervals of $\mathcal{T}_{n}^{(m)}$ by permutation of the labels. We prove an explicit formula, conjectured by F. Bergeron and the third author, for the character of the associated representation of $\mathfrak{S}_{n}$. In particular, the dimension of the representation, that is, the number of labelled $m$-Tamari intervals of size $n$, is found to be

$$
(m+1)^{n}(m n+1)^{n-2} .
$$

These results are new, even when $m=1$.

The form of these numbers suggests a connection with parking functions, but our proof is not bijective. The starting point is a recursive description of $m$-Tamari intervals. It yields an equation for an associated generating function, which is a refined version of the Frobenius series of the representation. The form of this equation is highly nonstandard: it involves two additional variables $x$ and $y$, a derivative with respect to $y$ and iterated divided differences with respect to $x$. The hardest part of the proof consists in solving it, and we develop original techniques to do so.
\end{abstract}

Keywords: Enumeration - Representations of $\mathfrak{S}_{n}$ - Lattice paths — Tamari lattices — Parking functions

\section{Introduction and main result}

An $m$-ballot path of size $n$ is a path on the square grid consisting of north and east unit steps, starting at $(0,0)$, ending at $(m n, n)$, and never going below the line $\{x=m y\}$. It is well-known that there are

$$
\frac{1}{m n+1}\left(\begin{array}{c}
(m+1) n \\
n
\end{array}\right)
$$

subm. to DMTCS (C) by the authors Discrete Mathematics and Theoretical Computer Science (DMTCS), Nancy, France 
such paths [DM47], and that they are in bijection with $(m+1)$-ary trees with $n$ inner nodes.

François Bergeron recently defined on the set $\mathcal{T}_{n}^{(m)}$ of $m$-ballot paths of size $n$ an order relation. It is convenient to describe it via the associated covering relation, exemplified in Figure 1.

Definition 1 Let $P$ and $Q$ be two $m$-ballot paths of size $n$. Then $Q$ covers $P$ if there exists in $P$ an east step $a$, followed by a north step $b$, such that $Q$ is obtained from $P$ by swapping $a$ and $S$, where $S$ is the shortest factor of $P$ that begins with $b$ and is a (translated) $m$-ballot path.

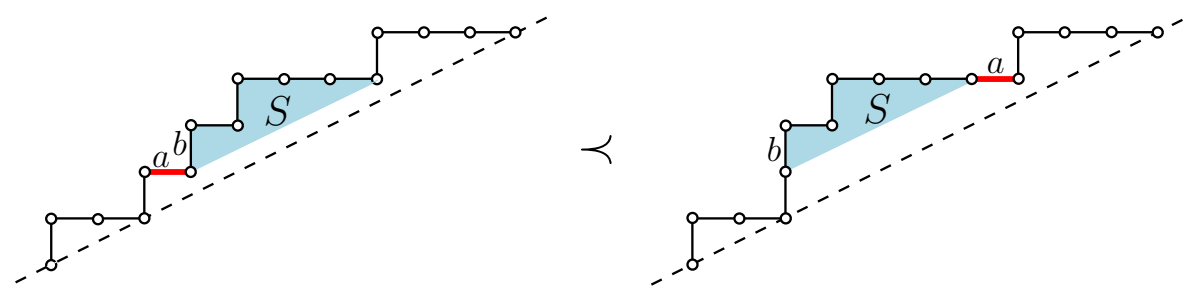

Fig. 1: The covering relation between $m$-ballot paths $(m=2)$.

It was shown in [BMFPR11] that this order endows $\mathcal{T}_{n}^{(m)}$ with a lattice structure, which is called the $m$-Tamari lattice of size $n$. When $m=1$, it coincides with the classical Tamari lattice [BB09, FT67, HT72, Knu06].

The interest in these lattices is motivated by their - still conjectural - connections with trivariate diagonal coinvariant spaces [BPRar, BMFPR11]. Some of them are detailed at the end of this introduction. In particular, it is believed that the representation of the symmetric group on these spaces is closely related to the representation of the symmetric group on labelled $m$-Tamari intervals. The aim of this paper is to characterize the latter representation, by describing explicitly its character.

So let us define this representation and state our main result. Let us call ascent of a ballot path a maximal sequence of consecutive north steps. An $m$-ballot path of size $n$ is labelled if the north steps are labelled from 1 to $n$, in such a way the labels increase along ascents (see the upper paths in Figure 2). These paths are in bijection with $(1, m, \ldots, m)$-parking functions of size $n$, in the sense of [SP02, Yan01]: the function $f$ associated with a path $Q$ satisfies $f(i)=k$ if the north step of $Q$ labelled $i$ lies at abscissa $k-1$. Using the cycle lemma [Rio69], it is easy to prove that the number of labelled $m$-ballot paths of size $n$ is

$$
(m n+1)^{n-1} .
$$

Now an $m$-Tamari interval $[P, Q]$ is labelled if the upper path $Q$ is labelled. The symmetric group $\mathfrak{S}_{n}$ acts on labelled intervals of $\mathcal{T}_{n}^{(m)}$ by permuting labels, and then reordering them in each ascent (Figure 2). This is the representation that we study here. We call it the m-Tamari representation of $\mathfrak{S}_{n}$. Our main result is an explicit expression for the character $\chi_{m}$ of this representation, which was conjectured by Bergeron and the third author [BPRar].

Theorem 2 Let $\lambda=\left(\lambda_{1}, \ldots, \lambda_{\ell}\right)$ be a partition of $n$ and $\sigma$ a permutation of $\mathfrak{S}_{n}$ having cycle type $\lambda$. Then for the m-Tamari representation of $\mathfrak{S}_{n}$,

$$
\chi_{m}(\sigma)=(m n+1)^{\ell-2} \prod_{1 \leq i \leq \ell}\left(\begin{array}{c}
(m+1) \lambda_{i} \\
m \lambda_{i}
\end{array}\right) .
$$



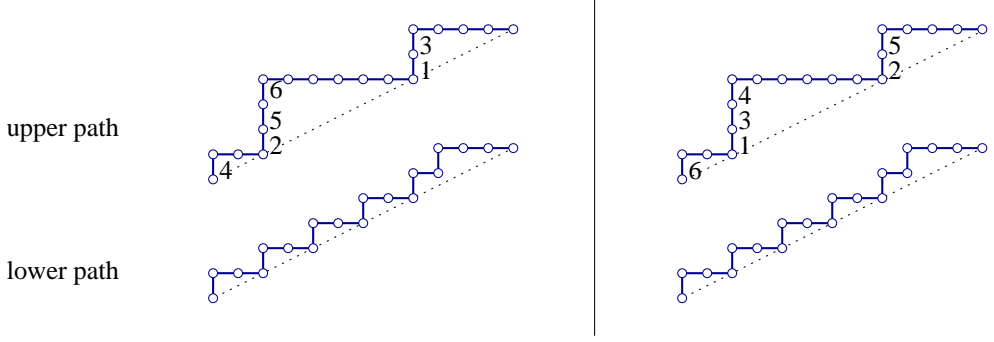

Fig. 2: A labelled 2-Tamari interval, and its image under the action of $\sigma=235614$.

Since $\mathfrak{S}_{n}$ acts by permuting labelled intervals, this is also the number of labelled $m$-Tamari intervals left unchanged under the action of $\sigma$.

In particular, the dimension of the representation, that is, the number of labelled $m$-Tamari intervals of size $n$, is

$$
\chi_{m}(\mathrm{id})=(m+1)^{n}(m n+1)^{n-2} .
$$

We were unable to find a bijective proof of these amazingly simple formulas. Instead, our proof uses generating functions and goes as follows. We introduce a generating function $F^{(m)}(t, p ; x, y)$ counting labelled intervals according to multiple parameters, where $p$ is an infinite sequence of variables $p_{1}, p_{2}, \ldots$, which can be thought of as power sums. We call this series the refined Frobenius series of the $m$-Tamari representation (Section 2). Then, we describe a recursive construction of intervals and translate it into a functional equation defining $F^{(m)}(t, p ; x, y)$ (Proposition 7, Section 3). The form of this equation is new to us, and its solution is the most difficult and original part of the paper. Due to lack of space, we only give the proof for $m=1$, after having explained the general principles of our approach, which are valid for all $m$ (Section 4). The complete version of this paper has appeared on the ArXiv [BMCPR12].

In the remainder of this section, we recall some of the conjectured connections between Tamari intervals and trivariate diagonal coinvariant spaces. They seem to parallel the (now proved) connections between ballot paths and bivariate diagonal coinvariant spaces, which have attracted considerable attention in the past 20 years [GH02, Hag03, Hag08, HL05, Hai94, Hai02, Loe03] and are still a very active area of research today, as shown by recent papers by Armstrong, Garsia, Haglund, Hicks, Loehr, Rhoades, Stout, Xin, Zabrocki... which we do not cite in details here for the sake of concision.

Let $X=\left(x_{i, j}\right)_{\substack{1 \leq i \leq k \\ 1 \leq j \leq n}}$. The diagonal coinvariant space $\mathcal{D} \mathcal{R}_{k, n}$ is defined as the quotient of the ring $\mathbb{C}[X]$ of polynomials in the coefficients of $X$ by the ideal $\mathcal{J}$ generated by constant-term free polynomials that are invariant under permuting the columns of $X$. For example when $k=2$, denoting $x_{1, j}=x_{j}$ and $x_{2, j}=y_{j}$, the ideal $\mathcal{J}$ is generated by constant-term free polynomials $f$ such that for all $\sigma \in \mathfrak{S}_{n}$,

$$
f(X)=\sigma(f(X)), \quad \text { where } \sigma(f(X))=f\left(x_{\sigma(1)}, \ldots, x_{\sigma(n)}, y_{\sigma(1)}, \ldots, y_{\sigma(n)}\right) .
$$

An $m$-extension of these spaces is of great importance here [GH96]. Let $\mathcal{A}$ be the ideal of $\mathbb{C}[X]$ generated by alternants under the diagonal action described above. There is a natural action of $\mathfrak{S}_{n}$ on the quotient space $\mathcal{A}^{m-1} / \mathcal{J} \mathcal{A}^{m-1}$. Let us twist this action by the $(m-1)^{\text {st }}$ power of the sign representation $\varepsilon$ : this gives rise to spaces

$$
\mathcal{D} \mathcal{R}_{k, n}^{m}:=\varepsilon^{m-1} \otimes \mathcal{A}^{m-1} / \mathcal{J} \mathcal{A}^{m-1},
$$


so that $\mathcal{D} \mathcal{R}_{k, n}^{1}=\mathcal{D} \mathcal{R}_{k, n}$. It is now a famous theorem of Haiman [Hai02, $\left.\mathrm{HHL}^{+} 05\right]$ that, as representations of $\mathfrak{S}_{n}$,

$$
\mathcal{D} \mathcal{R}_{2, n}^{m} \cong \varepsilon \otimes \operatorname{Park}_{m}(n)
$$

where $\operatorname{Park}_{m}(n)$ is the $m$-parking representation of $\mathfrak{S}_{n}$. This representation is defined as the action on labelled $m$-ballot paths ${ }^{(i)}$ that permutes the labels and then reorders them so that they increase in each ascent. The character of this representation, evaluated at a permutation of cycle type $\lambda=\left(\lambda_{1}, \ldots, \lambda_{\ell}\right)$ is $(m n+1)^{\ell-1}$. This formula, which generalizes (1), is easily proved using the cycle lemma.

In the case of three sets of variables, it is conjectured by Bergeron and the third author [BPRar] that, as representations of $\mathfrak{S}_{n}$,

$$
\mathcal{D} \mathcal{R}_{3, n}^{m} \cong \varepsilon \otimes \operatorname{Tam}_{m}(n),
$$

where $\operatorname{Tam}_{m}(n)$ is the representation of the symmetric group on labelled $m$-Tamari intervals that we study in this paper. The fact that the dimension of this space seems to be given by (3) is an earlier conjecture due to F. Bergeron (also observed for small values of $n$ by Haiman [Hai94] in the case $m=1$ ).

\section{The refined Frobenius series}

\subsection{Definitions and notation}

Let $\mathbb{L}$ be a commutative ring and $t$ an indeterminate. We denote by $\mathbb{L}[t]$ (resp. $\mathbb{L}[[t]]$ ) the ring of polynomials (resp. formal power series) in $t$ with coefficients in $\mathbb{L}$. If $\mathbb{L}$ is a field, then $\mathbb{L}(t)$ denotes the field of rational functions in $t$. This notation is generalized to polynomials, fractions and series in several indeterminates. We denote by bars the reciprocals of variables: for instance, $\bar{u}=1 / u$, so that $\mathbb{L}[u, \bar{u}]$ is the ring of Laurent polynomials in $u$ with coefficients in $\mathbb{L}$. The coefficient of $u^{n}$ in a Laurent polynomial $F(u)$ is denoted by $\left[u^{n}\right] F(u)$.

We use classical notation relative to integer partitions, which we recall briefly. A partition $\lambda$ of $n$ is a non-increasing sequence of integers $\lambda_{1} \geq \lambda_{2} \geq \cdots \geq \lambda_{\ell}>0$ summing to $n$. We write $\lambda \vdash n$ to mean that $\lambda$ is a partition of $n$. Each component $\lambda_{i}$ is called a part. For $i \geq 1$, the number of parts equal to $i$ in $\lambda$ is denoted by $m_{i}(\lambda)$. The cycle type of a permutation $\sigma \in \mathfrak{S}_{n}$ is the partition of $n$ whose parts are the lengths of the cycles of $\sigma$. This partition is denoted by $\lambda(\sigma)$. The number of permutations $\sigma \in \mathfrak{S}_{n}$ having cycle type $\lambda \vdash n$ equals $\frac{n !}{z_{\lambda}}$ where $z_{\lambda}:=\prod_{i \geq 1} i^{m_{i}(\lambda)} m_{i}(\lambda) !$.

We let $p=\left(p_{1}, p_{2}, \ldots\right)$ be an infinite list of independent variables, and for $\lambda$ a partition of $n$, we let $p_{\lambda}=p_{\lambda_{1}} \ldots p_{\lambda_{\ell(\lambda)}}$. The reader may view the $p_{\lambda}$ 's as the power sums in some ground set of variables. This point of view is not really needed in this paper, but it explains why we call refined Frobenius series our main generating function. Throughout the paper, we denote by $\mathbb{K}=\mathbb{Q}\left(p_{1}, p_{2}, \ldots\right)$ the field of rational fractions in the $p_{i}$ 's with rational coefficients.

Given a Laurent polynomial $P(u)$ in a variable $u$, we denote by $\left[u^{\geq}\right] P(u)$ the non-negative part of $P(u)$ in $u$, defined by

$$
\left[u^{\geq}\right] P(u)=\sum_{i \geq 0} P_{i} u^{i} \quad \text { if } \quad P(u)=\sum_{i \in \mathbb{Z}} P_{i} u^{i} .
$$

The definition is then extended by linearity to power series whose coefficients are Laurent polynomials in $u$. We define similarly the positive part of $P(u)$, denoted by $\left[u^{>}\right] P(u)$.

(i) which, as discussed above, are equivalent to parking functions 
We now introduce several series and polynomials which play an important role in this paper. They depend on two independent variables $u$ and $z$. First, we let $v \equiv v(u)$ be the following Laurent polynomial in $u$ :

$$
v=(1+u)^{m+1} u^{-m}
$$

We now consider the following series:

$$
V(u)=\sum_{k \geq 1} \frac{p_{k}}{k} v^{k} z^{k}
$$

It is is a formal power series in $z$ whose coefficients are Laurent polynomials in $u$ over the field $\mathbb{K}$. Finally we define the two following formal power series in $z$ :

$$
\begin{aligned}
L \equiv L(z, p) & :=\left[u^{0}\right] V(u)=\sum_{k \geq 1} \frac{p_{k}}{k}\left(\begin{array}{c}
(m+1) k \\
m k
\end{array}\right) z^{k}, \\
K(u) \equiv K(z, p ; u) & :=\left[u^{>}\right] V(u)=\sum_{k \geq 1} \frac{p_{k}}{k} z^{k} \sum_{i=m k+1}^{(m+1) k}\left(\begin{array}{c}
(m+1) k \\
i
\end{array}\right) u^{i-m k} .
\end{aligned}
$$

In our notation, we will often omit the dependence of series in $t, z$, and $p$, just keeping track, as above, of the variables $u, x$ and $y$, which play a special role in our equations.

\subsection{A refined theorem}

As stated in Theorem 2, the value of the character $\chi_{m}(\sigma)$ is the number of labelled intervals fixed under the action of $\sigma$, and one may see (2) as an enumerative result. Our main result is a refinement of (2) where we take into account two more parameters, which we now define. The first parameter is the number of contacts of the interval: A contact of an $m$-ballot path $P$ is a vertex of $P$ lying on the line $\{x=m y\}$, and a contact of an $m$-Tamari interval $[P, Q]$ is a contact of the lower path $P$. We denote by $c(P)$ the number of contacts of $P$.

By definition of the action of $\mathfrak{S}_{n}$ on $m$-Tamari intervals, a labelled interval $I=[P, Q]$ is fixed by a permutation $\sigma \in \mathfrak{S}_{n}$ if and only if $\sigma$ stabilizes the set of labels of each ascent of $Q$. Equivalently, each cycle of $\sigma$ is contained in the label set of an ascent of $Q$. If this holds, we let $r_{\sigma}(I)$ be the number of cycles of $\sigma$ occurring in the first ascent of $Q$ : this is our second parameter.

The main object we handle in this paper is a generating function for pairs $(\sigma, I)$, where $\sigma$ is a permutation and $I=[P, Q]$ is a labelled $m$-Tamari interval fixed by $\sigma$. In this series $F^{(m)}(t, p ; x, y)$, pairs $(\sigma, I)$ are counted by the size (variable $t$ ), the number $c(P)$ of contacts (variable $x$ ), the parameter $r_{\sigma}(Q)$ (variable $y$ ), and the cycle type of $\sigma$ (one variable $p_{i}$ for each cycle of size $i$ in $\sigma$ ). Moreover, $F^{(m)}(t, p ; x, y)$ is an exponential series in $t$. That is,

$$
F^{(m)}(t, p ; x, y):=\sum_{n \geq 0} \frac{t^{n}}{n !} \sum_{I=[P, Q]} \sum_{\sigma \in \operatorname{Stab}(I)} x^{c(P)} y^{r_{\sigma}(Q)} p_{\lambda(\sigma)},
$$

where the second and third sum are taken respectively on all labelled $m$-Tamari intervals $I$ of size $n$, and on all permutations $\sigma \in \mathfrak{S}_{n}$ fixing $I$. Note that when $(x, y)=(1,1)$, we have:

$$
F^{(m)}(t, p ; 1,1)=\sum_{n \geq 0} \frac{t^{n}}{n !} \sum_{I=[P, Q]} \sum_{\sigma \in \operatorname{Stab}(I)} p_{\lambda(\sigma)}=\sum_{n \geq 0} \frac{t^{n}}{n !} \sum_{\sigma \in \mathfrak{S}_{n}} \chi_{m}(\sigma) p_{\lambda(\sigma)}=\sum_{n \geq 0} t^{n} \sum_{\lambda \vdash n} \chi_{m}(\lambda) \frac{p_{\lambda}}{z_{\lambda}},
$$


since the value of character depends only on the cycle type (which justifies the notation $\chi_{m}(\lambda)$ ), and $\frac{n !}{z_{\lambda}}$ is the number of permutations of cycle type $\lambda$. Hence, in representation theoretic terms, $\left[t^{n}\right] F^{(m)}(t, p ; 1,1)$ is the Frobenius characteristic of the $m$-Tamari representation of $\mathfrak{S}_{n}$, also equal to $\sum_{\lambda \vdash n} c(\lambda) s_{\lambda}$, where $s_{\lambda}$ is the Schur function of shape $\lambda$ and $c(\lambda)$ is the multiplicity of the irreducible representation associated with $\lambda$ in the $m$-Tamari representation. For this reason, we call $F^{(m)}(t, p ; x, y)$ a refined Frobenius series.

Our most general result is a (complicated) expression of $F^{(m)}(t, p ; x, y)$, which becomes simple when $y=1$. We state here the result for $y=1$.

Theorem 3 Let $F^{(m)}(t, p ; x, y) \equiv F(t, p ; x, y)$ be the refined Frobenius series of the $m$-Tamari representation, defined by (7). Let $z$ and $u$ be two indeterminates, and write

$$
t=z e^{-m L} \quad \text { and } \quad x=(1+u) e^{-m K(u)},
$$

where $L \equiv L(z, p)$ and $K(u) \equiv K(z, p ; u)$ are defined by (5) and (6). Then $F(t, p ; x, 1)$ becomes a series in $z$ with polynomial coefficients in $u$ and the $p_{i}$, and this series has a simple expression:

$$
F(t, p ; x, 1)=(1+\bar{u}) e^{K(u)+L}\left((1+u) e^{-m K(u)}-1\right)
$$

with $\bar{u}=1 / u$. In particular, in the limit $u \rightarrow 0$, we obtain

$$
F(t, p ; 1,1)=e^{L}\left(1-m \sum_{k \geq 1} \frac{p_{k}}{k} z^{k}\left(\begin{array}{c}
(m+1) k \\
m k+1
\end{array}\right)\right) .
$$

Extracting the coefficient of $p_{\lambda} / z_{\lambda}$ (via Lagrange's inversion) shows that for a permutation $\sigma \in \mathfrak{S}_{n}$ of cycle type $\left(\lambda_{1}, \ldots, \lambda_{\ell}\right)$, the value of the $m$-Tamari character at $\sigma$ is given by (2).

Discovering the parametrization (8) of $t$ in terms of $z$ was easy using the conjectured value (2) of the character. Finding the parametrization of $x$ in terms of $u$ was difficult, but essential, since the variable $x$ is crucial in our approach (see (11)). Our expression of $F^{(m)}(t, p ; x, y)$ will appear in the complete version of the paper. When $m=1$, it takes a reasonably simple form.

Theorem 4 Let $F^{(1)}(t, p ; x, y) \equiv F(t, p ; x, y)$ be the refined Frobenius series of the 1-Tamari representation, defined by (7). Let $z$ and $u$ be two indeterminates, define $V(u), L$ and $K(u)$ by $(4-6)($ with $m=1)$ and set $t=z e^{-L}$ and $x=(1+u) e^{-K(u)}$. Then $F(t, p ; x, y)$ becomes a formal power series in $z$ with polynomial coefficients in $u$ and $y$, given by

$$
F(t, p ; x, y)=(1+u)\left[u^{\geq}\right]\left(e^{y V(u)-K(u)}-\bar{u} e^{y V(u)-K(\bar{u})}\right) .
$$

\section{Remarks}

1. It is easily seen that the case $y=1$ of (10) reduces to the case $m=1$ of (9).

2. When $p_{1}=1$ and $p_{i}=0$ for $i>1$, the only permutation that contributes is the identity. We are thus simply counting labelled 1-Tamari intervals, by their size (variable $t$ ), the number of contacts (variable $x$ ) and the size of the first ascent (variable $y$ ). We have $V(u)=v=(1+u)(1+\bar{u}), K(u)=z u$ and the extraction of the positive part in $u$ in (10) can be performed explicitly:

$$
\begin{aligned}
F(t, 1,0, \ldots ; x, y) & =(1+u)\left[u^{\geq}\right]\left(e^{y z v-z u}-\bar{u} e^{y z v-z \bar{u}}\right) \\
& =(1+u) e^{2 y z}\left(\sum_{0 \leq i \leq j} u^{j-i} \frac{z^{i+j} y^{i}(y-1)^{j}}{i ! j !}-\sum_{0 \leq j<i} u^{i-j-1} \frac{z^{i+j} y^{i}(y-1)^{j}}{i ! j !}\right) .
\end{aligned}
$$


When $x=1$, that is, $u=0$, the double sums in this expression reduce to simple sums, and the generating function of labelled Tamari intervals, counted by the size and the length of the first ascent, is expressed in terms of Bessel functions:

$$
\frac{F(t, 1,0, \ldots ; 1, y)}{e^{2 y z}}=\sum_{i \geq 0} \frac{z^{2 i} y^{i}(y-1)^{i}}{i !^{2}}-\sum_{j \geq 0} \frac{z^{2 j+1} y^{j+1}(y-1)^{j}}{(j+1) ! j !} .
$$

\section{A functional equation}

In this section, we describe a recursive construction of labelled $m$-Tamari intervals, and translate it into a functional equation satisfied by the generating function $F^{(m)}(t, p ; x, y)$.

We encode the north (resp. east) steps of a ballot path by the letters $\mathrm{N}$ and $\mathrm{E}$. We first recall a result that allows us to see the $m$-Tamari lattice as a sublattice of the standard 1-Tamari lattice (called Tamari lattice for short; similarly, a 1-ballot path is called below a ballot path).

Proposition 5 ([BMFPR11, Prop. 4]) Replacing each north step of an $m$-ballot path by a sequence of $m$ north steps induces an order preserving bijection between $\mathcal{T}_{n}^{(m)}$ and the sublattice of $\mathcal{T}_{n m}$ consisting of the paths that are larger than or equal to $\mathrm{N}^{m} \mathrm{E}^{m} \ldots \mathrm{N}^{m} \mathrm{E}^{m}$.

The functional equation of Proposition 7 below follows, although not in a straighforward manner, from a recursive description of Tamari intervals that can be found in [BMFPR11]. We say that a Tamari interval $I=[P, Q]$ is pointed if its lower path $P$ has a distinguished contact. Such a contact splits $P$ into two ballot paths $P^{\ell}$ and $P^{r}$, respectively located to the left and to the right of the contact. We use the notation $I=\left[P^{\ell} P^{r}, Q\right]$ to denote a pointed Tamari interval.

Proposition 6 ([BMFPR11]) Let $I_{1}=\left[P_{1}^{\ell} P_{1}^{r}, Q_{1}\right]$ be a pointed Tamari interval, and let $I_{2}=\left[P_{2}, Q_{2}\right]$ be a Tamari interval. Construct the ballot paths $P=\mathrm{N} P_{1}^{\ell} \mathrm{E} P_{1}^{r} P_{2}$ and $Q=\mathrm{N} Q_{1} \mathrm{E} Q_{2}$. Then $I=[P, Q]$ is a Tamari interval. Moreover, the mapping $\left(I_{1}, I_{2}\right) \mapsto I$ is a bijection between pairs $\left(I_{1}, I_{2}\right)$ formed of a pointed Tamari interval and a Tamari interval, and Tamari intervals I of positive size.

We do not give here the proof of the following proposition, but refer the reader to [BMCPR12].

Proposition 7 For $m \geq 1$, let $F^{(m)}(t, p ; x, y) \equiv F(x, y)$ be the refined Frobenius series of the m-Tamari representation, defined by (7). Then

$$
F(x, y)=\exp \left(y \sum_{k \geq 1} \frac{p_{k}}{k}\left(t x(F(x, 1) \Delta)^{(m)}\right)^{(k)}\right)(x),
$$

where $\Delta$ is the following divided difference operator

$$
\Delta S(x)=\frac{S(x)-S(1)}{x-1}
$$

and the powers $m$ and $k$ mean respectively that the operator $\Gamma: G(x, y) \mapsto F(x, 1) \cdot \Delta G(x, y)$ is applied $m$ times, and that the operator $G(x, y) \mapsto t x \cdot \Gamma^{m} G(x, y)$ is applied $k$ times.

Equivalently, $F(x, 0)=x$ and

$$
\frac{\partial F}{\partial y}(x, y)=\sum_{k \geq 1} \frac{p_{k}}{k}\left(t x(F(x, 1) \Delta)^{(m)}\right)^{(k)}(F(x, y)) .
$$




\section{Principle of the proof, and the case $m=1$}

\subsection{Principle of the proof}

Let us consider the functional equation (12), together with the initial condition $F(t, p ; x, 0)=x$. Perform the change of variables (8), and denote $G(z, p ; u, y) \equiv G(u, y)=F(t, p ; x, y)$. Then $G(u, y)$ is a series in $z$ with coefficients in $\mathbb{K}[u, y]$, satisfying

$$
\frac{\partial G}{\partial y}(u, y)=\sum_{k \geq 1} \frac{p_{k}}{k}\left(z(1+u) e^{-m(K(u)+L)}\left(\frac{u G(u, 1)}{(1+u) e^{-m K(u)}-1} \Delta_{u}\right)^{(m)}\right)^{(k)} G(u, y),
$$

with $\Delta_{u} H(u)=\frac{H(u)-H(0)}{u}$, and the initial condition

$$
G(u, 0)=(1+u) e^{-m K(u)} .
$$

This pair of equations defines $G(u, y) \equiv G(z, p ; u, y)$ uniquely as a formal power series in $z$. Indeed, the coefficient of $z^{n}$ in $G$ can be computed inductively from these equations (one first determines the coefficient of $z^{n}$ in $\frac{\partial G}{\partial y}$, which can be expressed, thanks to (13), in terms of the coefficients of $z^{i}$ in $G$ for $i<n$. Then the coefficient of $z^{n}$ in $G$ is obtained by integration with respect to $y$, using the initial condition (14)). Hence, if we exhibit a series $\tilde{G}(z, p ; u, y)$ that satisfies both equations, then $\tilde{G}(z, p ; u, y)=G(z, p ; u, y)$. We are going to construct such a series.

Let

$$
G_{1}(z, p ; u) \equiv G_{1}(u)=(1+\bar{u}) e^{K(u)+L}\left((1+u) e^{-m K(u)}-1\right) .
$$

Then $G_{1}(u)$ is a series in $z$ with coefficients in $\mathbb{K}[u]$, which, as we will see, coincides with $G(u, 1)$. Consider now the following equation, obtained from (13) by replacing $G(u, 1)$ by $G_{1}(u)$ :

$$
\frac{\partial \tilde{G}}{\partial y}(z, p ; u, y)=\sum_{k \geq 1} \frac{p_{k}}{k}\left(z(1+u) e^{-m(L+K(u))}\left(\frac{u G_{1}(u)}{(1+u) e^{-m K(u)}-1} \Delta_{u}\right)^{(m)}\right)^{(k)} \tilde{G}(z, p ; u, y)
$$

with the initial condition

$$
\tilde{G}(z, p ; u, 0)=(1+u) e^{-m K(u)} .
$$

Eq. (15) can be rewritten as

$$
\frac{\partial \tilde{G}}{\partial y}(u, y)=\sum_{k \geq 1} \frac{p_{k}}{k}\left(z(1+u) e^{-m K(u)} \Lambda^{(m)}\right)^{(k)} \tilde{G}(u, y)
$$

where $\Lambda$ is the operator defined by

$$
\Lambda(H)(u)=(1+u) e^{K(u)} \frac{H(u)-H(0)}{u} .
$$

Again, it is not hard to see that (17) and the initial condition (16) define a unique series in $z$, denoted $\tilde{G}(z, p ; u, y) \equiv \tilde{G}(u, y)$. The coefficients of this series lie in $\mathbb{K}[u, y]$. The principle of our proof can be described as follows. 
If we prove that $\tilde{G}(u, 1)=G_{1}(u)$, then the equation (15) satisfied by $\tilde{G}$ coincides with the equation (13) that defines $G$, and thus $\tilde{G}(u, y)=G(u, y)$. In particular, $G_{1}(z, p ; u)=$ $\tilde{G}(z, p ; u, 1)=G(z, p ; u, 1)=F(t, p ; x, 1)$, and Theorem 3 is proved.

\subsection{The case $m=1$}

Take $m=1$. We describe the three steps that, starting from (17), prove that $\tilde{G}(u, 1)=G_{1}(u)$. In passing, we establish the expression (10) of $F(t, p ; x, y)$ (equivalently, of $\tilde{G}(z, p ; u, y)$ ) given in Theorem 4.

\subsubsection{A homogeneous differential equation and its solution}

When $m=1$, the equation (17) defining $\tilde{G}(z ; u, y) \equiv \tilde{G}(u, y)$ reads

$$
\frac{\partial \tilde{G}}{\partial y}(u, y)=\sum_{k \geq 1} \frac{p_{k}}{k} z^{k}((1+u)(1+\bar{u}) \Omega)^{(k)} \tilde{G}(u, y)
$$

where $\bar{u}=1 / u$ and the operator $\Omega$ is defined by $\Omega H(u)=H(u)-H(0)$, with the initial condition

$$
\tilde{G}(u, 0)=(1+u) e^{-K(u)} .
$$

These equations imply that $\tilde{G}(-1, y)=0$. The following lemma provides us with a crucial symmetry property.

Lemma 8 For all $k \geq 0$ one has:

$$
((1+u)(1+\bar{u}) \Omega)^{(k)} \tilde{G}(u, y)=((1+u)(1+\bar{u}))^{k} \tilde{G}(u, y)-P_{k}(v, y)
$$

where $P_{k}(v, y) \in \mathbb{K}[y][[z]][v]$ is a polynomial in $v=(1+u)(1+\bar{u})$.

Proof: By induction on $k$. Alternatively, readers well acquainted with lattice path enumeration may view this lemma as a form of the reflection principle.

The quantity $P_{k}(v, y)$ is invariant under the substitution $u \mapsto \bar{u}$. This symmetry will enable us to eliminate some $a$ priori intractable terms in (18). Replacing $u$ by $\bar{u}$ in (18) gives

$$
\frac{\partial \tilde{G}}{\partial y}(\bar{u}, y)=\sum_{k \geq 1} \frac{p_{k}}{k} z^{k}((1+u)(1+\bar{u}) \Omega)^{(k)} \tilde{G}(\bar{u}, y)
$$

so that, applying Lemma 8 and using $v=(1+u)(1+\bar{u})$ we obtain:

$$
\frac{\partial}{\partial y}(\tilde{G}(u, y)-\tilde{G}(\bar{u}, y))=\sum_{k \geq 1} \frac{p_{k}}{k} z^{k}(1+u)^{k}(1+\bar{u})^{k}(\tilde{G}(u, y)-\tilde{G}(\bar{u}, y)) .
$$

This is now a homogeneous linear differential equation satisfied by $\tilde{G}(u, y)-\tilde{G}(\bar{u}, y)$. It is readily solved, and the initial condition (19) yields

$$
\tilde{G}(u, y)-\tilde{G}(\bar{u}, y)=(1+u)\left(e^{-K(u)}-\bar{u} e^{-K(\bar{u})}\right) e^{y V(u)},
$$

where $V(u)=\sum_{k \geq 1} \frac{p_{k}}{k} z^{k}(1+u)^{k}(1+\bar{u})^{k}$ as in (4). 


\subsubsection{Reconstruction of $\tilde{G}(u, y)$}

Recall that $\tilde{G}(u, y) \equiv \tilde{G}(z, p ; u, y)$ is a series in $z$ with coefficients in $\mathbb{K}[u, y]$. Hence, by extracting from the above equation the positive part in $u$ (as defined in Section 2.1), we obtain

$$
\tilde{G}(u, y)-\tilde{G}(0, y)=\left[u^{>}\right]\left((1+u)\left(e^{-K(u)}-\bar{u} e^{-K(\bar{u})}\right) e^{y V(u)}\right) .
$$

For any Laurent polynomial $P$, we have

$$
\left[u^{>}\right](1+u) P(u)=(1+u)\left[u^{>}\right] P(u)+u\left[u^{0}\right] P(u) .
$$

Hence

$$
\begin{aligned}
\tilde{G}(u, y)-\tilde{G}(0, y)=(1+u)\left[u^{>}\right]\left(e^{y V(u)}\left(e^{-K(u)}-\bar{u} e^{-K(\bar{u})}\right)\right) & \\
& +u\left[u^{0}\right]\left(e^{y V(u)}\left(e^{-K(u)}-\bar{u} e^{-K(\bar{u})}\right)\right) .
\end{aligned}
$$

Setting $u=-1$ in this equation gives, since $\tilde{G}(-1, y)=0$,

$$
-\tilde{G}(0, y)=-\left[u^{0}\right]\left(e^{y V(u)}\left(e^{-K(u)}-\bar{u} e^{-K(\bar{u})}\right)\right),
$$

so that finally,

$$
\begin{aligned}
\tilde{G}(u, y)= & (1+u)\left[u^{>}\right]\left(e^{y V(u)}\left(e^{-K(u)}-\bar{u} e^{-K(\bar{u})}\right)\right) \\
& +(1+u)\left[u^{0}\right]\left(e^{y V(u)}\left(e^{-K(u)}-\bar{u} e^{-K(\bar{u})}\right)\right) \\
= & (1+u)\left[u^{\geq}\right]\left(e^{y V(u)}\left(e^{-K(u)}-\bar{u} e^{-K(\bar{u})}\right)\right) .
\end{aligned}
$$

As explained in Section 4.1, $\tilde{G}(u, y)=G(u, y)$ will be proved if we establish that $\tilde{G}(u, 1)=G_{1}(u)$. This is the final step of our proof.

\subsubsection{The case $y=1$}

Eq. (21) completely describes the solution of (18). It remains to check that $\tilde{G}(u, 1)=G_{1}(u)$, that is,

$$
\tilde{G}(u, 1)=(1+\bar{u}) e^{K(u)+L}\left((1+u) e^{-K(u)}-1\right) .
$$

Let us set $y=1$ in (21). Using $V(u)=K(u)+L+K(\bar{u})$, this gives:

$$
\begin{aligned}
\tilde{G}(u, 1) & =(1+u)\left[u^{\geq}\right]\left(e^{V(u)-K(u)}-\bar{u} e^{V(u)-K(\bar{u})}\right) \\
& =(1+u)\left[u^{\geq}\right]\left(e^{L+K(\bar{u})}-\bar{u} e^{L+K(u)}\right) \\
& =(1+u) e^{L}\left(1-\bar{u} e^{K(u)}+\bar{u}\right)
\end{aligned}
$$

which coincides with (22). Hence $\tilde{G}(z, p ; u, y)=G(z, p ; u, y)=F(t, p ; x, y)$ (with the change of variables (8)), and Theorem 4 is proved, using (21). 


\subsection{A few words on the general case}

Assume $m>1$. Recall from Section 4.1 that we start from (17), and want to prove that $\tilde{G}(u, 1)=G_{1}(u)$. We consider the $m+1$ roots $u_{0}, \ldots, u_{m}$ of the equation $v(u)=v\left(u_{i}\right)$, where $v(u)=(1+u)^{m+1} \bar{u}^{m}$. They play a role similar to $u$ and $\bar{u}$ in Section 4.2. This enables us to obtain a generalization of (20), involving a linear combination of the $m+1$ series $\tilde{G}\left(u_{i}, y\right)$. The reconstruction of $\tilde{G}(u, y)$ and $\tilde{G}(u, 1)$ from this equation is possible, but difficult, and the expression it gives for $\tilde{G}(u, 1)$ did not allow us to prove directly that $\tilde{G}(u, 1)=G_{1}(u)$. Instead, we first proved that the generalization of (20) has a unique solution when $y=1$, and then checked that $G_{1}(u)$ is a solution. Proving uniqueness of the solution is difficult, and makes the proof for $m>1$ significantly longer than for $m=1$.

\section{Acknowledgements}

We are grateful to François Bergeron for advertising in his beautiful lectures various conjectures related to Tamari intervals. We also thank Éric Fusy and Gilles Schaeffer for interesting discussions on this topic.

\section{References}

[BB09] O. Bernardi and N. Bonichon. Intervals in Catalan lattices and realizers of triangulations. J. Combin. Theory Ser. A, 116(1):55-75, 2009.

[BMCPR12] M. Bousquet-Mélou, G. Chapuy, and L.-F. Préville-Ratelle. The representation of the symmetric group on $m$-Tamari intervals. Arxiv:1202.5925, 2012.

[BMFPR11] M. Bousquet-Mélou, É. Fusy, and L.-F. Préville-Ratelle. The number of intervals in the $m$-Tamari lattices. Electron. J. Combin., 2011. Arxiv 1106.1498, to appear.

[BPRar] B. Bergeron and L.-F. Préville-Ratelle. Higher trivariate diagonal harmonics via generalized Tamari posets. J. Combinatorics, to appear. Arxiv:1105.3738.

[DM47] A. Dvoretzky and Th. Motzkin. A problem of arrangements. Duke Math. J., 14:305-313, 1947.

[FT67] H. Friedman and D. Tamari. Problèmes d'associativité: Une structure de treillis finis induite par une loi demi-associative. J. Combinatorial Theory, 2:215-242, 1967.

[GH96] A. M. Garsia and M. Haiman. A remarkable $q, t$-Catalan sequence and $q$-Lagrange inversion. J. Algebraic Combin., 5(3):191-244, 1996.

[GH02] A. M. Garsia and J. Haglund. A proof of the $q, t$-Catalan positivity conjecture. Discrete Math., 256(3):677-717, 2002.

[Hag03] J. Haglund. Conjectured statistics for the $q, t$-Catalan numbers. Adv. Math., 175(2):319$334,2003$.

[Hag08] J. Haglund. The q,t-Catalan numbers and the space of diagonal harmonics, volume 41 of University Lecture Series. American Mathematical Society, Providence, RI, 2008. 
[Hai94] M. D. Haiman. Conjectures on the quotient ring by diagonal invariants. J. Algebraic Combin., 3(1):17-76, 1994.

[Hai02] M. Haiman. Vanishing theorems and character formulas for the Hilbert scheme of points in the plane. Invent. Math., 149(2):371-407, 2002.

$\left[\mathrm{HHL}^{+} 05\right] \quad J$. Haglund, M. Haiman, N. Loehr, J. B. Remmel, and A. Ulyanov. A combinatorial formula for the character of the diagonal coinvariants. Duke Math. J., 126(2):195-232, 2005.

[HL05] J. Haglund and N. Loehr. A conjectured combinatorial formula for the Hilbert series for diagonal harmonics. Discrete Math., 298(1-3):189-204, 2005.

[HT72] S. Huang and D. Tamari. Problems of associativity: A simple proof for the lattice property of systems ordered by a semi-associative law. J. Combin. Theory Ser. A, 13(1):7-13, 1972.

[Knu06] D. E. Knuth. The art of computer programming. Vol. 4, Fasc. 4. Addison-Wesley, Upper Saddle River, NJ, 2006. Generating all trees-history of combinatorial generation.

[Loe03] N. Loehr. Multivariate analogues of Catalan numbers, parking functions, and their extensions. PhD thesis, UCSD, San Diego, USA, 2003.

[Rio69] J. Riordan. Ballots and trees. J. Combinatorial Theory, 6:408-411, 1969.

[SP02] R. P. Stanley and J. Pitman. A polytope related to empirical distributions, plane trees, parking functions, and the associahedron. Discrete Comput. Geom., 27(4):603-634, 2002.

[Yan01] C. H. Yan. Generalized parking functions, tree inversions, and multicolored graphs. Adv. in Appl. Math., 27(2-3):641-670, 2001. 